Gerade so, wie die Lichtabsorption unter chemischer Verbindung sich ändert, wenn man weisses, wasserfreies Kurpfersulfat mit Wasser zusammenbringt, so ändert sich hier die elektrische „Farbe" des Pyridins durch den Wasserzusatz, und gerade so wie beim Kupfersulfat kann man auch hier geneigt sein, diese elektrische "Farbenänderung" durch Hydratbildung zu erklären, wie schon Drude beim Acetaldehyd in Gemisch mit Wasser (l. c.) geschlossen hat.

(Eingegraugen: I5. Juni.)

\title{
ZUR KENNTNIS DER ELEKTROLYTISCHEN REDUKTION AROMATISCHER NITROKÖRPER ZU AMINEN ${ }^{1}$.

\author{
Von Dr. A. Chilesotti.
}

(Mitteilung ans dem Flektrochemischen Iaboratoriunr der Königl. Sächs. Technischen Hochschule zu Dresden.)

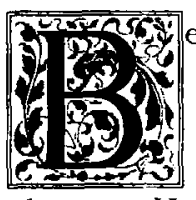
ei der elektrolytischen Reduktion aromatischer Nitrokörper war es bisher nur gelungen, die Bedingungen festzustellen, unter denen man glatt zu Hydroxylamin-Abkömmlingen 2) (bezw. Amidophenolen ${ }^{3}$ ) oder zu den Azoxy-4), den Azo-5) oder Hydrazoverbindungen 5) (bezw. Benzidinen ${ }^{4}$ )) gelangen konnte, die gleiche Möglichkeit bestand aber für dic elektrolytische Gewinnung der Amine bisher nicht. Diese Lücke ist nunmehr durch die schönen Verfahren ausgefüllt, welche seitens der Firma C. F. Boehringer \& Söhneb), sowie vor kurzen von Elbs und Silbermann ${ }^{7}$ ) bekannt gegeben sind. Jene setzt zu dem gedachten Zwecke der sauren Lösung oder Suspension des Nitrokörpers kleine Mengen von Salzen des Zinns, Kupfers, Quecksilbers oder des dreiwertigen Eisens oder Chroms $z u$, diese benutzen statt der früher von Elbs angewandten Zinkkathode jetzt mit ausgezeichnetem Erfolge eine Bleikathode gegenüber einem nicht allzu stark sauren Elektrolyten.

Nachdem ich durch kurze Referate in der

1) Die hier mitgeteilte Untersuchung hat Herr Dr. Chilesotti auf meine Veranlassung ausgefübrt, da ich wünschte, die wichtige elektrolytische Herstellung aromatischer Anine alsbald den in meinem Laboratorium auszuführenden Uebungsaufgaben anzureihell. Nach der italieuischen Niederschrift des Verfassers habe ich den vorliegenden Auszug angefertigt. Foerster.

2) Haber, Diese Zeitschr. 5, 77.

3) Gattermann, Ber. d. D. Chen. Ges. 26, 1844; 27, 1927.

4) Löb, diese Zeitschr. 7, $33+$ und 336 .

5) Elbs, diese Zeitschr. 5, 108, 7, 133 und 14I.

6) D. R. - P. II6942 und I 7007 vom 24. Mai, bezw. 1o. April I8gg.

7) E1bs und Silberman11, diese Zeitsclrr. 7, $5^{89}$.
Zeitschrift für angewandte Chemie ${ }^{1}$ ) von den Grundzügen der Bochringerschen Arbeitsweise Kenntnis genommen hatte, habe ich einige Versuche angestellt, um diese Verfahren etwas naher kennen zu lernen, und dabei mich von ihrem ausserordentlich hohen Werte überzeugt, den ja inzwischen auch $\mathrm{Häussermann}{ }^{2}$ ) hervorgehoben hat. Obgleich meine Erfahrungen die Angaben der genannten Patente bestätigen, glaube ich doch, dass vielleicht demjenigen, der im Laboratorium die Verfahren ausführen will, einige noch etwas nähere Angaben uber die Versuchsbedingungen gelegen kommen können, und halte es daher bei der grossen Wichtigkeit des Gegenstandes nicht für ganz überflüssig, meine Versuche hier wiederzugeben, umsomehr, als ich noch einige die Theorie der Erscheinung betreffende Beobachtungen in Anschluss hieran mitteiten möchte. Die Ausführungsweise meiner elektrolytischen Versuche, welche, wie gesagt, wesentlich für das Laboratorium gedacht ist, habe ich in mehrfachen Einzelheiten selbständig bestimmt, da der Wortlaut der Patentschriften mir zur Zeit der Ausführung der Mehrzahl meiner Versuche noch nicht zugänglich war; daraus erklären sich gewisse Abweichungen gegen spezielle Angaben der Patentnehmer. Es ist aber vielleicht nicht uninteressant, dass die für das Gelingen der Boehringerschen Verfahren bestimmenden Bedingungen keine allzu eng umgrenzten sind.

Die Ausführung der Versuche war die folgende: Als Anodenraum diente eine $6,5 \mathrm{~cm}$ weite Thonzelle, in welche $\mathrm{s} 80$ bis $200 \mathrm{ccm}$ - -...- -

I) Zeitschr. 'f. angew. Chem. Igor, 64 und $1_{42}$.

2) Häussermanu, Zeitschr. f. angew. Chemie $1901,3^{80}$. 
neunprozentige Schwefelsăure gegeben wurden. Die Anode bestand meist aus einem cylindrisch aufgerollten Bleiblech; ihre Stromzuführung war durch den die Anode verschliessenden Kork geführt, welcher gleichzeitig auch ein Ableitungsrohr trug fär die - bei Anwendung von Salzsăure im Kathodenraum stets chlorhaltigen Gase. Diese Zelle stand in einem $10,5 \mathrm{~cm}$ weiten Becherglase und war von der aus Nickeldrahtnetz bestehenden, einen Cylindermantel bildenden Kathode umgeben. Zwischen dieser und der Thonzelle konnte ein aus einem Glasstabe gebogener, ringformiger Rührer mit der Hand auf - und abbewegt werden.

Die Kathodenlauge muss für den vorliegenden Zweck freie Säure, Salzsäure oder Schwefelsäure, enthalten. $\mathrm{Da}$ diese aber bekanntlich bei hoher Konzentration die an Platinkathoden zunächst aus aromatischen Nitrokörpern entstehenden Hydroxylaminabkömmlinge in chlorierte Aniline, bezw. Amidophenole umlagern und ich einen ahnlichen Einfluss auch bei meinen Versuchen fürchtete, so habe ich mich bemüht, mit einem möglichst geringen Saureüberschuss auszukommen. Man thut gut, um am Schluss des Versuches eine klare Losung zu erhalten, die Säuremenge so zu bemessen, dass die Kathodenlauge nach der Elektrolyse durch freie Saure noch mindestens 0,7 - bis 0,8 -fach normal ist; aber auch die doppelte Menge dieser Säure konnte ohne Schaden angewandt werden.

In solchen verdünnten wässerigen Säurelösungen sind aromatische Nitrokörper nur sehr beschränkt löslich. Stellt man aber eine Emulsion her, deren Zustandekommen ich durch voraufgehendes Lossen der Nitroverbindung in etwas Alkohol begünstigte, oder bei den bei gewöhnlicher Temperatur festen Nitrokörpern durch Erwärmen des Elektrolyten über ihren Schmelzpunkt bewirkte, so erfolgte bei Gegenwart kleiner Mengen eines Zinn - oder Kupfersalzes die elektrolytische Reduktion doch äusserst leicht. Oft unterbleibt wăhrend des grösseren Teiles der Elektrolyse jede Spur einer Wasserstoffentwicklung auch ohne Umrühren, und es genügt meist, nur im letzten Abschnitt der Elektrolyse den Ruhrer in Thätigkeit zu setzen. Alsdann verläuft die Reduktion bis nahezu zum Ende ohne jede Wasserstoffentwicklung und liefert, wie die Patentschriften angeben, eine hăufig den theo- retiscben Werten sehr nahekommende Stromund Materialausbeute. Wichtig hierfür ist es, dass Säure- und Wassermenge so bemessen sind, dass wahrend der Reduktion keine unlöslichen Stoffe (basische Metallsalze, schwer losliche Chlorhydrate der Basen) sich auf der Kathode niederschlagen. Vermeidet man dies, so ergiebt die Elektrolyse, wenigstens bei Gegenwart von Zinnchlorür, die wasserhelle, metallfreie Lösung der salz- oder schwefelsauren Salze der erwarteten Amine; bei Gegenwart von Kupfer- oder Eisensalzen enthălt die Lösung meist gefärbte, zum Teil als Oele suspendierte Nebenprodukte in kleiner Menge.

Die benutzte Stromdichte belief sich auf Io bis I $2 \mathrm{Amp} / \mathrm{qdm}$; da die Kathode mit $190 \mathrm{qcm}$ einseitiger Oberflache eintauchte, bedeutet das eine Stromstärke von 19 bis 22 Amp. Diese liess sich während des Versuches leicht konstant halten, so dass die angewandte Strommenge aus der Versuchsdauer zu ermitteln war; meist wurde ein kleiner Stromüberschuss in den Elektrolyten gesandt zur Sicherstellung der volligen Reduktion der Nitroverbindung. Die Badspannung betrug niemals mehr als 5,0 bis 5,6 Volt.

Das Elektrolysiergefäss stand in einem grossen Gefässe mit kaltem Wasser. Etwa ro Minuten nach Einschaltung des Stromes hatte im Elektrolyten, der hohen Stromdichte zufolge, die Temperatur 40 bis $45^{\circ}$ erreicht und stieg nun langsam bis auf etwa $50^{\circ}$; bei den meisten Versuchen herrschte also eine zwischen 40 und $5^{\circ}$ liegende Temperatur. Wollte man bei etwa $20^{\circ}$ arbeiten, so gelang dies dadurch, dass als Anode ein spiralig aufgewundenes, starkes Bleirohr benutzt wurde, durch welches 9 bis ro ${ }^{0}$ kaltes Leitungswasser strömte.

Die Menge der gewonnenen Basen kann, wenigstens wenn Zinnchlorür benutzt wird, durch Wägen des Abdampfrückstandes der Kathodenlauge ermittelt werden. Ich habe jedoch fast immer, um mich von der Reinheit der erhaltenen Basen zu überzeugen, die saure Losung, nach Abdestillieren des Alkohols, alkalisch gemacht und die Basen mit Wasserdampf abgeblasen. Nach Abscheidung aus dem Destillat waren die Körper sofort völlig rein.

Die Ergebnisse einiger Versuche seien in der folgenden Uebersicht zusammengestellt: 
J. Reduktion von Nitrobenzol.

a) Bei Gegenwart von Zinnchloriir.

\begin{tabular}{|c|c|c|c|c|c|c|}
\hline $\begin{array}{l}\text { Versuch } \\
\mathrm{Nr} \text {. }\end{array}$ & Zusammensetzung der Kathodenksung & $\begin{array}{l}\text { Mittlere } \\
\text { Versuchs- } \\
\text { temperatur }\end{array}$ & $\begin{array}{l}\text { Angewandte } \\
\text { Strommenge } \\
\text { in Ampère- } \\
\text { Stunden }\end{array}$ & Erhaltenes Produkt & $\begin{array}{l}\text { Material- } \\
\text { ausbeute }\end{array}$ & $\begin{array}{l}\text { Strom- } \\
\text { ausbeute }\end{array}$ \\
\hline $\mathbf{I}$ & $\begin{array}{c}20 \mathrm{~g} \text { Nitrobenzol in } 30 \mathrm{ccm} \text { Alko- } \\
\text { hol, } 250 \mathrm{ccm} \text { Wasser mit I } \mathrm{g} H C l \\
\text { und I } \mathrm{g} S n C l_{\mathrm{s}}, 2 \mathrm{H}_{\mathrm{g}} \mathrm{O}\end{array}$ & $45^{0}$ & 26,5 & $12,76 \mathrm{~g}$ Anilin & $84,41 / 0$ & $83.5^{\prime \prime \prime}$ \\
\hline 2 & wie bei Versuch Nr. I & $20^{\prime \prime}$ & 28,5 & $1 \mathrm{I}, 4$ & $75 \cdot 4 \%$ & $66,9 \%$ \\
\hline 3 & $\begin{array}{l}4^{\circ} \mathrm{g} \text { Nitrobenzol in } 30 \mathrm{ccm} \text { Alko- } \\
\text { hol, } 25 \text { occm Wasser mit } 22 \mathrm{~g} \mathrm{HCl} \\
\text { und I } \mathrm{g} S n \mathrm{Cl}_{2}, 2 \mathrm{H}_{2} \mathrm{O}\end{array}$ & $47^{0}$ & 53,5 & 23,1, & $7^{6,4^{10}} \cdot$ & $76,4^{1 / 0}$ \\
\hline 4 & wie bei Versuch Nr. 3 & $2 I^{\prime \prime}$ & 55,9 & 25,8 & $85,4 \%$ & $80,9 \%$ \\
\hline \multicolumn{7}{|c|}{ b) Bei Gegenwart von Kupfersalzen. } \\
\hline 5 & $\mid \begin{array}{c}20 \mathrm{~g} \text { Nitrobenzol in } 30 \mathrm{ccm} \text { Alko- } \\
\text { hol, 250 ccm Wasser mit i } 7,6 \mathrm{~g} \mathrm{HCl} \\
\text { und I g CuCl } \mathrm{Cl}_{2}, 2 \mathrm{H}_{2} \mathrm{O}\end{array}$ & $55^{\circ}$ & 28,4 & $12,6 \mathrm{~g}$ Anilin & $83,1^{\prime \prime}$ & $75.1^{11 !}$ \\
\hline 6 & $\mid \begin{array}{c}20 \mathrm{~g} \text { Nitrobenzol in } 30 \text { ccm Alkohol, } \\
250 \text { cem Wasser mit } 23,6 \mathrm{~g} \mathrm{H} \mathrm{SO}_{4} \\
\text { und I,5 g CuSO }, 5 \mathrm{H}_{2} \mathrm{O}\end{array}$ & $45^{\prime \prime}$ & 28,2 & I I,8 & $7^{8,} 3^{\prime \prime}$ & $70,5^{\circ} \cdot$ \\
\hline
\end{tabular}

2. Reduktion substituicrter Nitrobenzole (stets bei Gegeuwart von Zinnchlorïr).

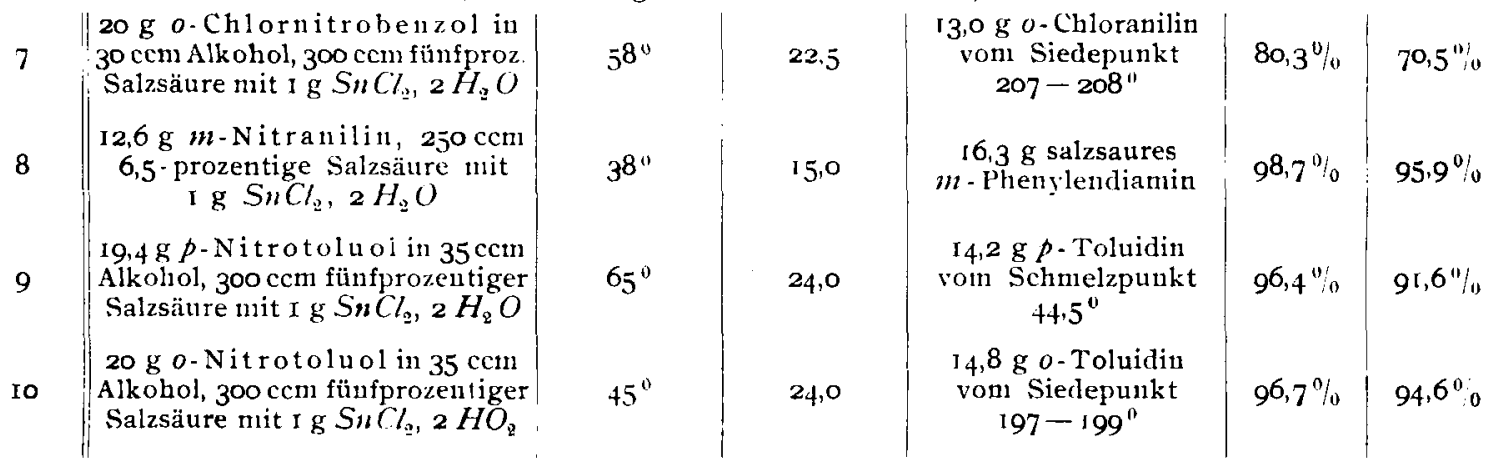

Diese Versuche zeigen, wic ausserordentlich wertvoll die Verfahren der Firma C. F. Boehringer \& Söhne zur elektrolytischen Erzeugung aromatischer Amine sind.

Im einzelnen ergab sich, dass bei Anwesenheit von Zinnchlorür unter sonst gleichen Umständen die Reduktion des Nitrobenzols etwas leichter erfolgt als wenn Kupfersalze, zumal Kupfersulfat, in der Lösung vorhanden sind. Man hat es jedoch stets in der Hand, durch Vermehrung des Säurezusatzes oder Erhohung der Temperatur oder energisches Umrühren eine unter gewissen Umständen etwas träge, also unter Wasserstoffentwicklung verlaufende Reduktion zu beschleunigen.

Auf die hohe theoretische Bedeutung der Boehringerschen Erfahrungen ist in den genannten Patentschriften, sowic in ihrer Be- sprechung in dieser Zeitschrift schon hingewiesen worden. In Bestätigung der in den Patent. schriften geausserten Ansichten möchte ich zunächst darauf hinweisen, dass bei allen obigen Versuchen sich das Nickeldrahtnetz mit schwam. migem Zinn oder Kupfer bedeckt zeigte. Benutz: man eine so vorbereitete Kathode, so kann mar mit ihr auch in einem ursprünglich von Metallsalz freien Elektrolyten ähnliche Resultate er. halten, wie sie bei den vorstehenden Versuchen erzielt wurden. Dies entspricht der Boeh. ringerschen Angabe, dass man bei Gegenwart von Zinn sehr grosse Mengen Nitrobenzol in demselben Elektrolyten ohne Erneuerung des Zusatzes von Zinnsalz reduzieren kann. Geht man aber von einem blanken Kupferdrahtnetz. als Kathode aus und unterlässt den Zusatz von Kupfersalz zum Elektrolyten, so ist die Ueber- 
fahrung des Nitrobenzols in Anilin auch bei grossem Stromüberschuss eine ganz unvollkommene.

Dies rührt daher, dass zum Zustandekommen des Vorganges stets abwechselnd Metall kathodisch sich niederschlagen und dann wieder auflösen muss. Wahrend fein verteiltes Kupfer dies genügend schrell thut, ist das glatte Kupferdrahtnetz hierzu nicht im stande.

Für den hier gedachten Reduktionsmechanismus ist wohl folgender Versuch beweisend: Reduziert man bei Gegenwart von Zinnsalz Nitrobenzol, so enthält die lösung kein Zinn mehr, wenn alles Nitrobenzol in Anilin verwandelt ist. Setzt man jetzt, ohne den Strom zu unterbrechen, wieder eine bleine Menge Nitrobenzol dem Elektrolyten zu, so enthält dieser auch sofort wieder Zinnsalz, welches erst mit Vollendung der Reduktion verschwindet. Man darf also zur Deutung der Erscheinungen katalytische Einflüsse der Metalle nicht heranziehen.

Zur Aufklärung der Art ihrer Wirksamkeit bei der in Rede stehenden Reaktion ging ich, einer Anregung von Herrn Dr. Müller folgend, von der Ueberlegung aus, dass doch auch in den vorliegenden Fällen, wie stets bei der elektrolytischen Reduktion aromatischer Nitrokörper, zunächst eine Hydroxylaminverbindung, aus Nitrobenzol also Phenylhydroxylamin, entstehen müsste. Eine solche konnte, wie das Hydroxylamin selbst '), in Berührung mit oxydierbaren Metallen von diesen mit grosser Geschwindigkeit zu der zugehörigen Aminverbindung reduziert werden. Zur Entscheidung dieser Frage wurden die folgenden Versuche angestellt.

Von meinen oben mitgeteilten Elektrolysen möchte ich den bei Gegenwart von Kupfersalzen ausgeführten ein erbohtes theoretisches Interesse zuschreiben. Sie zeigen, dass, wenu man die Elbssche Beobachtung, nach der Nitrobenzol an Zinkkathoden zu Anilin reduziert wird, durch das hier besonders hohe Kathodenpotential deutet, damit das Wesen der Erscheinung nicht getroffen ist. Denn man sieht, ganz dieselbe Reduktion kann an einem Metalle erfolgen, das wie Kupfer die Kathode auf einem verhältnismässig tiefen Potential zu halten vermag.

Kupfer selbst vermag nun auf rein chemischem

1) Vergl. F. Müller, Zeitschr. f. anorg. Chem. 26, I (IgOI).
Wege Nitrobenzol nur mit höchst unbedeutender Geschwindigkeit zu Anilin zu reduzieren. Als ... in möglichster Anlehnung an die bei der Elektrolyse herrschenden Bedingungen - $5 \mathrm{~g}$ Nitrobenzol in $6 \mathrm{ccm}$ Alkohol langsam und unter lebhaftem Schütteln in $90 \mathrm{ccm}$ fünfprozentiger, $45^{\circ}$ warmer Salzsäure eintropfen gelassen wurden, in welcher sich $15 \mathrm{~g}$ elektrolytisch hergestellten Kupferschwamms befanden, war eine nennenswerte Reaktion nicht zu bemerken. Nach etwa einer halben Stunde wurde, ohne das Kupfer zu entfernen, zum Sieden erbitzt, der Alkohol abdestilliert, dann das Nitrobenzol und schliesslich, nach Alkalizusatz, etwa entstandenes Anilin mit Wasserdampf abgeblasen. Es wurde fast alles Nitrobenzol wiedergewonnen und nur $5,3 \%$ der an Anilin theoretisch zu erwartenden Ausbeute erhalten.

Ganz anders verläuft der Prozess, wenn Kupferschwamm auf Phenylhydroxylamin einwirkt: eine Lösung von $4,4 \mathrm{~g}$ dieses Korpers in $6 \mathrm{ccm}$ Alkohol wurde bei $45^{\circ}$ in $60 \mathrm{ccm}$ fanfprozentiger Salzsäure getropft, in welcher $5 \mathrm{~g}$ Kupferschwamm verteilt waren. Es tritt lebhafte Reduktion ein, Kupfer geht in Lơsung, und gegen Ende der eine Viertelstunde beanspruchenden Operation nimmt die Lösung eine olivenbraune Fărbung an, welche man auch bei der Elektrolyse des Nitrobenzols in Gegenwart von Kupfer stets bemerkt. Wurde unmittelbar nach beendetem Prozess filtriert, alkalisch gemacht und im Dampfstrom abgeblasen, so ging nur Anilin aber, und es wurden 4,02 g seines Chlorhydrates, d. h. $77 \%$ der theoretischen Ausbeute, an Anilin gewonnen.

Dieser Versuch ist an sich in der von mir verfolgten Richtung noch nicht ganz schlussig, denn das gefundene Anilin könnte ja zu einem mehr oder weniger grossen Teil anderen Einflüssen als denjenigen des Kupfers entstammen, was bei einer so ausserordentlich reaktionsfahigen Substanz, wie Phenylhydroxylamin ist, nicht unwahrscheinlich wäre. Zunächst könnte dieses durch den benutzten Alkohol reduziert worden sein. Dabei müsste allerdings nach einer von mir bestätigten Beobachtung $\mathrm{B}$ a m bergers Azoxybenzol auftreten, wovon aber bei obigem Versuche auch nicht eine Spur zu finden war. Nichtsdestoweniger habe ich denselben ohne Alkoholzusatz wiederholt. Es wurden in $90 \mathrm{ccm}$ fünfprozentiger, wässeriger Salzsäure von $45^{\circ}$, 
in welcher $9 \mathrm{~g}$ Kupferschwamm aufgeschlämmt waren, $5 \mathrm{~g}$ Phenylhydroxylamin im Laufe einer Viertelstunde unter lebhaftem Umschwenken eingetragen, wobei wieder Kupfer lebhaft in Lösung ging. Nun wurde schnell filtriert und das Filtrat $(140 \mathrm{ccm}$ ) zur Beseitigung unveränderten Phenylhydroxylamins mit $37 \mathrm{~g}$ konzentrierter Schwefelsäure versetzt und aufgekocht. Alsdann wurde mit Alkali übersăttigt, im Dampfstrom abgeblasen und das Destillat, mit Salzsäure versetzt, zur Trockne gebracht. Es wurden $3,8 \mathrm{I} \mathrm{g}$ eines Chlorhydrates erhalten, dessen Basis, in Freiheit gesetzt, vollståndig bei $18 \mathrm{I}^{0}$ überging. Es war also auch jetzt Anilin, und zwar in einer Ausbeute von $64 \%$, entstanden.

Diese Anilinbildung kann nur der Wirkung des Kupfers zugeschrieben werden. Denn als I5 g Phenylhydroxylamin genau der entsprechenden Behandlung wie bei dem eben beschriebenen Versuche unterworfen wurden, mit dem einzigen Unterschiede, dass der Zusatz des Kupfers ganz in Wegfall kam, wurde zwar auch ein basisches Oel erhalten, das aber jetzt erst bei $207^{\circ} \mathrm{zu}$ sieden begann, und dessen Hauptteil zwischen $215^{\circ}$ und $224^{0}$ überging und im Destillat zu weissen Krystallen vom Schmelzpunkt $70^{\circ}$ erstarrte. Dieses Oel enthält also kein Anilin, sondern besteht im wesentlichen aus $p$-Chloranilin und daneben aus etwas $o$-Chloranilin, welche der Einwirkung der Salzsäure auf Phenylhydroxylamin ihre Entstehung verdanken.

Nach diesen Versuchen darf man mit Sicherheit schliessen, dass Phenylhydroxylamin durch feinverteiltes Kupfer bei Gegenwart von Salzsäure $\left.{ }^{1}\right)$ sehr schnell zu Anilin reduziert wird.

Andere, weniger edle Metalle als Kupfer, dürften mindestens ebenso schnell wie dieses reduzierend auf Phenylhydroxylamin einwirken. Auch eine saure Ferrosalzlösung, welche auf Nitrobenzol kaum einwirkt, lässt aus Phenylhydroxylamin reichlich Anilin entstehen. Doch

1) Auch ohne Säure, z. B. beim Zusammenreiben mit wenig Wasser, traten Kupfer und Phenylhydroxylanin in lebhafte Reaktion, können also überhaupt wohl nicht nebeneinander bestehen. Unter starker Temperaturerhöhung verflüssigt sich die Masse; es bildet sich ein Oel, welches aber nur zum Teil aus Anilin besteht. Die nähere Verfolgung dieses interessanten Vorganges habe ich unterlassen, da sie von meinem Ziele abführte. verlăuft der Prozess hier anscheinend ziemlich verwickelt.

Nach den im vorangehenden gemachten Feststellungen bedurfte nur noch folgender Punkt der Klarlegung. Aus Habers schönen Untersuchungen wissen wir, dass bei der elektrolytischen Reduktion des Nitrobenzols zunächst Nitrosobenzol entsteht, aber sofort weitcre Umwandlung zu Phenylhydroxylamin, bezw. durch Umsetzung mit diesem zu Azoxybenzol erleidet. Es war immerhin nicht ausgeschlossen, dass auch dieser durch sein hohes Oxydationspotential ausgezeichnete Korper energisch mit oxydierbaren Metallen reagiert. Daher wurden $4,35 \mathrm{~g}$ Nitrosobenzol in $25 \mathrm{ccm}$ Alkohol gelöst, im Verlauf einer Viertelstunde zu $75 \mathrm{ccm}$ fünfprozentiger Salzsäure und Io g Kupferpulver bei $45^{\circ}$ zugetropft. Nach vollendeter Operation roch die Lösung noch nach Nitrosobenzol, und gab bei der Dampfdestillation Azoxybenzol, Azobenzol und Anilin, letzteres in einer $37 \%$ der Theorie entsprechenden Menge. Die Reduktion durch Kupfer und Salzsăure verläuft also nicht so schncll, dass sie nicht dem noch übrigen Ausgangskörper erlaubte, mit seinen Reduktionsprodukten in bekannter Weise zusammenzutreten, was auch deshalb schon wahnscheinlich ist, da an einer Kathode, an der Nitrobenzol reduziert wird, nach $\mathrm{Haber}$ Nitrosobenzol uberhaupt nur in Spuren auf eine messbare Zeit bestehen kann. Da bei meinen Elektrolysen weder Azoxy- noch Azoverbindungen auftraten, kann also für die Deutung der Erscheinungen von der Einwirkung der Metalle auf das Nitrosobenzol abgesehen werden.

Nach diesen Versuchen darf der Verlauf der Vorgänge bei dem Boehringerschen Verfahren zur elektrolytischen Reduktion aromatischer Nitrokörper zu Aminen, zunächst bei Gegenwart von Kupfer-oder Ferrisalzen, folgendermaassen aufgefasst werden: Der Strom reduziert anfangs, wie er es auch bei Abwesenhcit der genannten Salze thut, den Nitrokörper zum Ary'hydroxylamin und schlägt zugleich schwammiges Kupfer nieder, oder erzeugt Ferrosalz. Diese reduzieren nun ihrerseits, und zwar, wie die beschriebenen Versuche zeigen, auch während der Elektrolyse auf rein chemischem Wege das Arylhydroxylamin mit grosser Geschwindigkeit zum Amin. Dabei gehen sie wieder in das 
Kupfer-Ion, oder das Ferri-Ion über und bieten sich dem Strome aufs neue dar. Ob dieser nun auch nebenher bei dem durch das Kupfer-, bezw. das Ferrosalz gegebenen Kathodenpotential primär die Reduktion des Arylhydroxylamins zum Amin bewirken kann, bleibt noch eine offene, jedenfalls olne weiteres nicht zu verneinende Frage.

Die Vorstellung, dass bei der elektrolytischen Erzeugung aromatischer Amine eine elektrolytische Vorarbeit des Stromes und eine chemische Nacharbeit des durch einen Teil der ersteren erzeugten Metalles, bezw, niedrig wertigen lons stattfindet, lässt sich für die Gegenwart von Kupfer- oder Ferrisalz durch Versuche begründen. Das Gleiche ist nicht thunlich für positivere Metallkathoden, z. B. für Zinn, Blei oder Zink, oder für ein so starkes Reduktionsmittel, wie es die Chromo-lonen sind. Denn diese Körper reduzieren, zum Unterschiede von Kupfer oder Ferro-Ion, mit beträchtlicher Geschwindigkeit auch unmittelbar aromatische Nitroverbindungen. Darum darf man noch nicht annehmen, dass bei Gegenwart der Salze unedlerer Metalle die gesamte elektrolytische
Arbeit des Stromes etwa im Niederschlagen der genannten Metalle besteht und diese dann rein chemisch die ganze Reduktion besorgen. Vielmehr ist auch hier durchaus dem Strom Gelegenheit gegeben, primär Arylhydroxylamin zu bilden. Die niedergeschlagenen Metalle können nun sowohl diese Verbindung, wie die Nitroverbindung, auf chemischem Wege reduzieren; dabei dürften sie die erstere bevorzugen, da sie deren Reduktion offenbar mit der grosseren Geschwindigkeit auszufähren vermogen. Die Vorgänge bei der elektrolytischen Reduktion aromatischer Nitroverbindungen in Gegenwart von Kupfersalz einerseits oder Zinnsalz anderseits sind also höchstens graduell verschieden.

Die Verfahren von C. F. B o ehringer \& Söhne und von Elbs und Silbermann sind so wesensverwandt, dass ich glaube, sie beide in theoretischer Hinsicht in das bisher erfahrungsgemås festgestellte Schema der elektrolytischen Reduktion aromatischer Nitroverbindungen durch obige Versuche eingeschlossen zu haben.

Dresden, 18. Juni I90ı.

(Eingegangen: I9. Juni.)

\section{R E P E R T OR I U M.}

\section{ALLGEMEINE ELEKTROCHEMIE.}

Für das Erwachen elektrochemischer Interessen in England hat vor kurzem die Gründung der Zeitschrift ,The Electro-Chemist and Metallurgist" Zeugnis gegeben. Eine zweite, noch erfreulichere Frucht dieser Bestrebungen finden wir in der Mai-Nummer dieser Zeitschrift von $\mathrm{R}$. S. Hutton beschrieben, nämlich die Gründung und Einrichtung des ersten elektrochemischen Laboratoriums Englands an der Victoria-Universitat in Owens College, Manchester. Das neue Institut ist den physikalischen Instituten angegliedert, die unter Leitung von Professor Schuster stehen. Als "demonstrator" und "assistant lecturer" für Elektrochemie wirkt dort Hutton, M. Sc. Der Beschreibung nach ist das neue Institut recht zweckmässig cingerichtet und mit grossen Mitteln an Dynamomaschinen und Akkumulatoren für Ströme bis zu I 000 Ampère ausgestattet. Mit Recht ist Gewicht darauf grelegt, dass die rein chcmische Ausrüstung nichts zu wünschen übrig lässt. Einige Bilder bringen die Räıme des Laboratoriums zur Anschauungr. Wünsclien wir der neuen Pflanzstätte unserer Wissenschaft bestes Gedeihen!
R. A.
Die Wirkungen der Gleichstromschwankungen der elektrischen Schwingungen und der Elektroinduktionsströme auf eine in einem konstanten Felde stehende Magnetnadel, bezw. einen Induktor aus weichem Eisen. M. Hornemann. Ann. d. Physik 4, 481-512. Separatabdruck vom Verf. Ein Hinweis auf die Arbeit muss genügen.

H. D.

Das elektrochemische Aequivalent des Kupfers und Silbers. Th. W. Richards, E. Collins und G. W. Heimrod, Zeitschr. f. phys. Chemic 82, $321-347$ (I9oo). Die Ver- . suche des Verf. ergaben für Kupfer im wesentlichen diesclben Resultate, die Foerster und Seidel (Zeitschr. f. anorg. Chemie 14, I06 bis I45; vergl. diese Zeitschr. 5, 508) gefunden hatten, und die sich kurz folgendermaassen zusammenfassen lassen. 1. Kupfer löst sich langsam in angesäuertem Kupfersulfat, auch unter Wasserstoffatmosphäre. Ungefähr scheint der Verlust an elcktrolytisch reinem Kupfer der Oberfläche proportional zu sein. 2. Da die Wirkung einer 0,2 n.-sauren Lösung wenig verschieden von der einer 0,02 n.-sauren Lösung ist, können 